\title{
Exploring Factors Associated with Cyclist Injury Severity in Vehicle-Electric Bicycle Crashes Based on a Random Parameter Logit Model
}

\author{
Fei Ye $\mathbb{D},{ }^{1,2}$ Changshuai Wang $\mathbb{D},{ }^{3}$ Wen Cheng $\mathbb{D},{ }^{1,2}$ and Haoxue Liu ${ }^{1}$ \\ ${ }^{1}$ School of Transportation Engineering, Chang'an University, Xi'an 710064, China \\ ${ }^{2}$ School of Rail Transit, Zhejiang Institute of Communications, Hangzhou 311112, China \\ ${ }^{3}$ School of Transportation, Southeast University, Nanjing 210096, China \\ Correspondence should be addressed to Fei Ye; yefei@zjvtit.edu.cn
}

Received 23 February 2021; Revised 25 June 2021; Accepted 20 July 2021; Published 31 July 2021

Academic Editor: Antonino Vitetta

Copyright ( 2021 Fei Ye et al. This is an open access article distributed under the Creative Commons Attribution License, which permits unrestricted use, distribution, and reproduction in any medium, provided the original work is properly cited.

Electric bicyclists are vulnerable road users and play an important role in traffic safety. The focus of this research is on analyzing cyclists' injury severity in vehicle-electric bicycle collisions. It is an exploratory analysis that was conducted based on samples obtained from video data provided by the police of Xi' an China. Three types of severity include fatal, injury, and property-damageonly (PDO). A random parameter logit (RPL) model was specified to gain more insights into factors related to the injury severity level, including human behaviors, vehicle characteristics, roadway attributes, and environmental conditions. Some factors not included in previous research were introduced into this study, especially precrash behaviors of drivers and cyclists. The direct pseudo-elasticity effects of variables were compared to investigate the stability of individual parameter estimates on the severity categories. The results indicated that variables that significantly increment the probability of fatal accidents were as follows: driver violation behaviors (speeding, red-light violation, driving in the opposite direction), cyclist violation behaviors (speeding, redlight violation), day of time (nighttime), visibility restrictions (fixed obstacles), and vehicle type (larger bus, small truck, and larger truck). Based on these findings, we suggested measures such as strengthening law enforcement by installing cameras, implementing zero tolerance for cyclist violations, promoting education by completing training courses for cyclists, and enhancing traffic safety awareness through educational activities. The research results can provide a theoretical basis for formulating strategies to improve cyclist safety.

\section{Introduction}

Electric bicycle has become a popular travel tool due to the advantages of mobility, flexibility, and environmental protection [1]. However, it also brings along traffic safety problems. The rate of electric-bicycle-related casualties was significantly increased [2]. Meanwhile, road traffic and bicycle-related casualties have decreased. Electric bicycle is gradually replacing the role of traditional bicycle in travel tools and traffic crashes [3]. The rapid increase in the number of deaths from electric bicycles has led to a steady rise in the overall proportion of vulnerable road users' deaths. However, despite the safety issues brought about by electric bicycles, literature review found that most of the studies about cyclist injury severity were focused on traditional bicycles. It is necessary to have a better understanding of the relationship between the injury severity of collisions and the risk factors, so as to provide a theoretical support for the traffic management department to formulate control measures. In fact, the electric bicycle safety issues have been studied in the United States, Canada, Europe, Japan, and some other countries [4,5]. Electric bicycle has its own characteristics. The influencing factors of cyclist injury severity are also different from factors that affect conventional bicycle users [6]. Under the background of the rapid outbreak of electric bicycles in China, the factors and degree of influence may be different from developed countries. Therefore, it is necessary to investigate the factors that lead to 
collisions involving electric bicycles and analyze cyclist injury severity.

This research aims to optimize the traditional discrete choice model into the random parameter logit (RPL) model for quantitative analysis of the vehicle-electric bicycle collisions by considering the unobserved heterogeneity among predictor variables. The vehicle-electric bicycle collisions in Xi'an China were taken as a sample. Accidents were classified into three severity levels: fatal, injury, and propertydamage-only (PDO). Then, we use a RPL model to determine the main factors related to the cyclist injury severity in vehicle-electric bicycle collisions by considering human behaviors, vehicle characteristics, roadway attributes, and environmental conditions. The direct pseudo-elasticity effects of variables on injury severity categories were also introduced and compared. Finally, some countermeasures for the authorities to alleviate traffic accident problems were provided according to the results.

In addition to considering some common factors as in previous articles, this paper also focuses on the precrash collision avoidance and violation behaviors of drivers and cyclists as factors affecting the severity of the accident. The influence of human behaviors on injury severity is analyzed in depth, which is different from previous studies. The results show that this consideration has a good effect.

\section{Literature Review}

According to statistics, the deaths and injuries suffered by cyclists have increased significantly in recent years, which is related to the rapid growth of electric bicycles. Efforts have been made to explore the various significant factors that contribute to injury severity level of e-cyclists. Many studies have investigated factors associated with injury severity of e-cyclists when collided with vehicles. Regarding rider demographic characteristics, some results have shown that age and gender are the most relevant factors to the severity of accidents. Yuan et al. [3] found that older cyclists and younger drivers were more likely to be involved in fatal crashes based on analyzing collisions between electric bicycles and cars in Beijing, China.

In addition to age and gender, the riding experience can also significantly affect the injury severity outcomes. Young and inexperienced cyclists are more likely to be involved in a crash due to risky behaviors, while older cyclists tend to be involved in fatal crashes because of their slower perception and action speed and poorer physical function [3, 4, 7-9]. Novice cyclists are associated with higher risk of accidents and injuries [10, 11]. Fyhri et al. [12] found that female riders had a higher risk of accidents, which could be attributed to their unfamiliarity with electric bicycles.

The violation behaviors will significantly increase the severity, such as speeding, disobeying traffic signals, riding on the motorized lane, wrong-direction riding, red-light running, and riding without helmet [13]. Studies [10, 14, 15] found that e-bike cyclists with red-light running would suffer more serious injury. Based on China's in-depth accident research data, the relationship between cyclist casualty level and the impact speed in vehicle-electric bicycle collisions were quantitatively investigated [9]. Qian et al. [10] found that red-light running, drunk riding, illegally carrying adults, turning without signaling, illegal occupation of motorways, and factors such as electric bicycle type were possible risk factors for electric bicycle accidents. The speeding behavior would contribute to higher probability of fatal crashes $[8,9]$. Not wearing a helmet could cause more serious damage $[6,16]$. In terms of accident fault assignment, poor-educated and male riders were more likely to commit violations in e-bike accidents, and e-bike riders were more likely to be at fault in urban areas [17].

In studies related to the injury severity of traffic accidents, vehicle type is found to be a significant cause. Several studies have demonstrated the different effects of vehicle type on the severity level. However, the effect of vehicle type was rarely considered in the study about electric vehicles involved in accidents [7]. The larger momentum and the presence of blind areas of large vehicles might lead to drivers' inability to see cyclists. There was significant correlation between the type of accident and injury severity $[15,18]$. Similarly, crash location was also an important factor affecting the outcomes of accidents, which was considered in other types of accidents, such as collision between vehicles. However, in the current literature review, this factor was not considered in the study of accidents involving electric bicycles, probably because of the difficulty of data acquisition. In addition, factors such as road type, lighting conditions, weather conditions, and visibility restrictions, and so on, would contribute to the crashes $[6,19,20]$. Relevant research believed that the causes of vehicle-electric bicycle collisions were diverse [21], and the consequences were also different.

The above discussion shows that some influencing factors have relatively similar effects on the injury severity of different subjects. However, in different studies, there are also some factors showing contradictory effects. There are several reasons that may lead to this situation, including the unobserved heterogeneity in the data, the differences in methods, the insufficient number of observations, and the differences in space and time.

The data from questionnaires and records from police or hospital were usually used $[5-8,10,16]$. From the perspective of accident severity at the macro level, some scholars believed that the video data obtained by the driving recorders or cameras were relatively more realistic in causation analysis [22]. Considering the difficulty in reproducing the process of the accident, with the help of dash cam video data, the collision avoidance behaviors, environments, and road conditions before the accident could be clearly grasped. Ito et al. [23] used dash cam data to compare and analyze the different factors that affect vehicle-bicycle collisions and approaching collisions. The collisions would not be avoided even if the drivers had taken some evasive actions. Rea et al. [24] reviewed dash cam videos on YouTube ${ }^{\mathrm{TM}}$ that recorded moose-vehicle interactions and built a logit regression model, and found that "vehicle deceleration" was a significant variable to explain whether a moose would collide with a vehicle. Guo et al. [25] evaluated the safety impacts of powered two wheelers on a shared roadway by using automated computer video analysis. Min and Ando [26] 
analyzed the video and GPS data of the driving recorder, found that dangerous driving events were closely related to the driver's age and gender, the environment and driving time, and the driver's mental state. The video data obtained by the driving recorders or cameras had covered the information of the driver, vehicle, road, and environment involved in the accidents. It is a kind of data that is more authentic than the questionnaire results or records.

However, the violation and collision avoidance behaviors of drivers and cyclists before the accidents are not captured in the records of the police and hospital; this leads to insufficient understanding of the relationship between impact factors and injury severity of cyclists in previous research. Moreover, there are still differences in considering the impact of various factors on the crash severity. Therefore, it is valuable to conduct an in-depth, detailed, and comprehensive analysis of the factors affecting the crash severity. The results of this study will provide useful information to formulate effective policies, especially for the management to improve the safety situation of e-bikes involved in transportation in China.

\section{Data Preparation}

The injury severity of a traffic crash is defined by the level of injury sustained by the most severely injured occupant. In an accident, the cyclists are often in roles of vulnerable participants that sustain the most serious injury. Therefore, the severity of accidents involving electric bicycles is measured by the injury severity of the cyclist. Although there are many ways to classify the level of injury severity, the standard classification into fatal, injury, and property-damage-only crashes is widely used in the analysis of accident severity [27]. Statistics found that there was no reason to be alarmed by the general property-damage-only accidents, as only the casualties were reported and then recorded by the police. It presents some difficulties to accident severity analysis. The videos provide unique source of data for road traffic managers in exploring what happened in the moments just before the collision. Therefore, the data used in this study are obtained from the vehicle-electric bicycle crash video provided by the traffic police department of Xi'an, China, between 2017 and 2019.

Efforts have been devoted to building the dataset. We invited six students of transportation engineering major from two classes (three in each group). The checking task did not start officially until the students were trained to ensure that they were familiar with the task. Also, they were required to be objective and accurate in the process. Each video was viewed 15-20 times by the two groups. The variables were identified, which were hypothesized that may influence the likelihood of injury severity level. All factors were categorized into human behaviors, vehicle characteristics, roadway attributes, and environmental conditions. The human behaviors analyzed in the study include collision avoidance and violation behaviors of drivers and cyclists. Vehicle characteristics include vehicle type and crash type. Roadway attributes include road type, crash location, number of lanes, etc. Environmental conditions include the day of time, weather conditions, visibility restrictions, and the electric bicycle visibility time. Objectively speaking, it was difficult to confirm whether the cyclists died or not in the accident just by checking the video. We compared the results with the police reports as a remedy. Based on the literature review, we extracted 15 aspects of accident-related factors that were consistently observable in each video. The behaviors of drivers or cyclists ("accelerating," "decelerating," or "lane change," etc.) were determined by detecting the trend of vehicle speed relative to environmental surroundings (highway lines, roadside reflectors, trees, etc.). We did not attempt to calculate the deceleration or acceleration rate; we simply recorded whether or not the vehicle slowed or accelerated. In particular, for the factor of "visibility restrictions," we roughly estimated the types of visibility restrictions through the positional geometric relationship between vehicle and e-bike in the videos. The remaining three continuous independent variables were "Number of lanes in direction of an electric bicycle," "Number of lanes in direction of a vehicle," and "Electric bicycle visibility time." We defined "Electric bicycle visibility time" as the number of seconds that the bicyclist was visible from his or her first appearance in drivers' sight until he or she was struck by the vehicle. Some of the videos showed information strips with a clock that we read to for calculation purposes. If no clock was observed embedded in the video, we used a stop watch to measure the time. We roughly estimated the number of lanes in different directions based on the lane lines. Although in some cases it was too dark to determine the number of lanes, the width of the vehicle provides sufficient basis to estimate the number of lanes. When the results identified by the two groups of students were inconsistent with each other, they were asked to recheck the accident video jointly until the final result was confirmed.

After data cleaning, the original data were organized in the accident database and then divided into three categories based on the criteria for modeling the severity of accident [28] and similar studies [27]: PDO (127 or 10.6\%), injury (900 or $74.7 \%$ ), and fatal (174 or $14.5 \%$ ). Variables that have similar definitions were carefully examined and combined, which was consistent with many existing studies. Table 1 presents the statistical description of injury severity level frequency and percentage distribution by 12 categorical independent variables. A majority (74.9\%) of the cyclists in the sample were suffering from injury as a result of the crash. The road type of crashes in the sample turned out to be mostly $(81.1 \%)$ on urban roads; this was consistent with the results of the study by Wang et. al [17]. Table 1 also reveals that about $61.4 \%$ of the electric bicycles in the sample were hit in the side by vehicles and about $62.4 \%$ of the crashes happened at intersections.

\section{Methodology}

Many researchers have studied the injury severity of traffic participants in recent years. For instance, Hu et al. [7] explored the related risk factors of injuries caused by e-bike and bicycle crashes in Hefei, Anhui. Wang et al. [8] used the 
TABLE 1: Description of injury severity level frequency and percentage distribution by the explanatory variables.

\begin{tabular}{|c|c|c|c|c|c|c|c|c|}
\hline \multirow{3}{*}{$\begin{array}{l}\text { Variable description } \\
\text { Severity }\end{array}$} & \multicolumn{8}{|c|}{ Injury severity } \\
\hline & \multicolumn{2}{|c|}{ Total } & \multicolumn{2}{|c|}{$\mathrm{PDO}$} & \multicolumn{2}{|c|}{ Injury } & \multicolumn{2}{|c|}{ Fatal } \\
\hline & $N=1201$ & $100 \%$ & $N=127$ & $10.6 \%$ & $N=900$ & $74.9 \%$ & $N=174$ & $14.5 \%$ \\
\hline \multicolumn{9}{|c|}{ Day of time } \\
\hline Daytime* & 887 & $73.9 \%$ & 109 & $85.8 \%$ & 653 & $72.6 \%$ & 125 & $71.8 \%$ \\
\hline Nighttime & 211 & $17.6 \%$ & 9 & $7.1 \%$ & 165 & $18.3 \%$ & 37 & $21.3 \%$ \\
\hline Dawn/dusk & 103 & $8.6 \%$ & 9 & $7.1 \%$ & 82 & $9.1 \%$ & 12 & $6.9 \%$ \\
\hline \multicolumn{9}{|c|}{ Weather conditions } \\
\hline Sunny* & 945 & $78.7 \%$ & 110 & $86.6 \%$ & 684 & $76.0 \%$ & 151 & $86.8 \%$ \\
\hline Rainy/snowy & 140 & $11.7 \%$ & 13 & $10.2 \%$ & 116 & $12.9 \%$ & 11 & $6.3 \%$ \\
\hline Cloudy & 116 & $9.7 \%$ & 4 & $3.1 \%$ & 100 & $11.1 \%$ & 12 & $6.9 \%$ \\
\hline \multicolumn{9}{|c|}{ Road type } \\
\hline Urban* & 974 & $81.1 \%$ & 106 & $83.5 \%$ & 718 & $79.8 \%$ & 150 & $86.2 \%$ \\
\hline Rural & 182 & $15.2 \%$ & 19 & $15.0 \%$ & 142 & $15.8 \%$ & 21 & $12.1 \%$ \\
\hline Mountainous & 45 & $3.7 \%$ & 2 & $1.6 \%$ & 40 & $4.4 \%$ & 3 & $1.7 \%$ \\
\hline \multicolumn{9}{|c|}{ Crash location } \\
\hline Straight* & 381 & $31.7 \%$ & 37 & $29.1 \%$ & 285 & $31.7 \%$ & 59 & $33.9 \%$ \\
\hline Curved & 71 & $5.9 \%$ & 9 & $7.1 \%$ & 57 & $6.3 \%$ & 5 & $2.9 \%$ \\
\hline Intersection & 749 & $62.4 \%$ & 81 & $63.8 \%$ & 558 & $62.0 \%$ & 110 & $63.2 \%$ \\
\hline \multicolumn{9}{|c|}{ Crash type } \\
\hline Hit the stationary vehicle* & 135 & $11.2 \%$ & 18 & $14.2 \%$ & 104 & $11.6 \%$ & 13 & $7.5 \%$ \\
\hline Head-on collision with vehicle & 247 & $20.6 \%$ & 25 & $19.7 \%$ & 192 & $21.3 \%$ & 30 & $17.2 \%$ \\
\hline Hit in the side by vehicle & 738 & $61.4 \%$ & 69 & $54.3 \%$ & 555 & $61.7 \%$ & 114 & $65.5 \%$ \\
\hline Hit in the rear-end by vehicle & 81 & $6.7 \%$ & 15 & $11.8 \%$ & 49 & $5.4 \%$ & 17 & $9.8 \%$ \\
\hline \multicolumn{9}{|c|}{ Visibility restrictions } \\
\hline Without restriction* & 714 & $59.5 \%$ & 93 & $73.2 \%$ & 496 & $55.1 \%$ & 125 & $71.8 \%$ \\
\hline Fixed obstacle & 198 & $16.5 \%$ & 8 & $6.3 \%$ & 168 & $18.7 \%$ & 22 & $12.6 \%$ \\
\hline Moving vehicle & 289 & $24.1 \%$ & 26 & $20.5 \%$ & 236 & $26.2 \%$ & 27 & $15.5 \%$ \\
\hline \multicolumn{9}{|c|}{ Whether the cyclist has a lookout motion? } \\
\hline No* & 813 & $67.7 \%$ & 83 & $65.4 \%$ & 590 & $65.6 \%$ & 140 & $80.5 \%$ \\
\hline Yes & 388 & $32.3 \%$ & 44 & $34.6 \%$ & 310 & $34.4 \%$ & 34 & $19.5 \%$ \\
\hline \multicolumn{9}{|c|}{ Collision avoidance behaviors of drivers } \\
\hline None* & 152 & $12.7 \%$ & 6 & $4.7 \%$ & 95 & $10.6 \%$ & 51 & $29.3 \%$ \\
\hline Accelerating & 10 & $0.8 \%$ & 0 & $0.0 \%$ & 9 & $1.0 \%$ & 1 & $0.6 \%$ \\
\hline Decelerating & 802 & $66.8 \%$ & 101 & $79.5 \%$ & 637 & $70.8 \%$ & 64 & $36.8 \%$ \\
\hline Lane change & 28 & $2.3 \%$ & 4 & $3.1 \%$ & 16 & $1.8 \%$ & 8 & $4.6 \%$ \\
\hline Decelerating and lane change & 209 & $17.4 \%$ & 16 & $12.6 \%$ & 143 & $15.9 \%$ & 50 & $28.7 \%$ \\
\hline \multicolumn{9}{|c|}{ Violation behaviors of drivers } \\
\hline None* & 627 & $52.2 \%$ & 83 & $65.4 \%$ & 488 & $54.2 \%$ & 56 & $32.2 \%$ \\
\hline Speeding & 206 & $17.2 \%$ & 14 & $11.0 \%$ & 129 & $14.3 \%$ & 63 & $36.2 \%$ \\
\hline Illegal lane change & 83 & $6.9 \%$ & 6 & $4.7 \%$ & 68 & $7.6 \%$ & 9 & $5.2 \%$ \\
\hline Red-light violation & 21 & $1.7 \%$ & 3 & $2.4 \%$ & 11 & $1.2 \%$ & 7 & $4.0 \%$ \\
\hline Driving in the opposite direction & 60 & $5.0 \%$ & 2 & $1.6 \%$ & 45 & $5.0 \%$ & 13 & $7.5 \%$ \\
\hline Making a turn without turning light & 73 & $6.1 \%$ & 11 & $8.7 \%$ & 53 & $5.9 \%$ & 9 & $5.2 \%$ \\
\hline Illegal U-turn & 131) & $10.9 \%$ & 8 & $6.3 \%$ & 106 & $11.8 \%$ & 17 & $9.8 \%$ \\
\hline & Collision av & dance be & aviors of & clists & & & & \\
\hline None* & 446 & $37.1 \%$ & 32 & $25.2 \%$ & 315 & $35.0 \%$ & 99 & $56.9 \%$ \\
\hline Accelerating & 15 & $1.2 \%$ & 5 & $3.9 \%$ & 6 & $0.7 \%$ & 4 & $2.3 \%$ \\
\hline Decelerating & 225 & $18.7 \%$ & 42 & $33.1 \%$ & 166 & $18.4 \%$ & 17 & $9.8 \%$ \\
\hline Lane change & 140 & $11.7 \%$ & 5 & $3.9 \%$ & 113 & $12.6 \%$ & 22 & $12.6 \%$ \\
\hline Decelerating and lane change & 375 & $31.2 \%$ & 43 & $33.9 \%$ & 300 & $33.3 \%$ & 32 & $18.4 \%$ \\
\hline & Viola & behavic & of cyc & & & & & \\
\hline None* & 345 & $28.7 \%$ & 39 & $30.7 \%$ & 242 & $26.9 \%$ & 64 & $36.8 \%$ \\
\hline Speeding & 204 & $17.0 \%$ & 33 & $26.0 \%$ & 142 & $15.8 \%$ & 29 & $16.7 \%$ \\
\hline Illegal lane change & 110 & $9.2 \%$ & 15 & $11.8 \%$ & 82 & $9.1 \%$ & 13 & $7.5 \%$ \\
\hline Red-light violation & 231 & $19.2 \%$ & 9 & $7.1 \%$ & 187 & $20.8 \%$ & 35 & $20.1 \%$ \\
\hline Driving in the opposite direction & 163 & $13.6 \%$ & 19 & $15.0 \%$ & 135 & $15.0 \%$ & 9 & $5.2 \%$ \\
\hline Turning without turning light & 90 & $7.5 \%$ & 3 & $2.4 \%$ & 70 & $7.8 \%$ & 17 & $9.8 \%$ \\
\hline Illegal occupation of the motor vehicle lanes & 58 & $4.8 \%$ & 9 & $7.1 \%$ & 42 & $4.7 \%$ & 7 & $4.0 \%$ \\
\hline
\end{tabular}


TABLE 1: Continued.

\begin{tabular}{|c|c|c|c|c|c|c|c|c|}
\hline \multirow{2}{*}{ Variable description } & \multicolumn{8}{|c|}{ Injury severity } \\
\hline & \multicolumn{2}{|c|}{ Total } & \multicolumn{2}{|c|}{ PDO } & \multicolumn{2}{|c|}{ Injury } & \multicolumn{2}{|c|}{ Fatal } \\
\hline \multicolumn{9}{|c|}{ Vehicle type } \\
\hline Passenger car* & 1056 & $87.9 \%$ & 121 & $95.3 \%$ & 838 & $93.1 \%$ & 97 & $55.7 \%$ \\
\hline Larger bus & 14 & $1.2 \%$ & 1 & $0.8 \%$ & 8 & $0.9 \%$ & 5 & $2.9 \%$ \\
\hline Small truck & 33 & $2.7 \%$ & 1 & $0.8 \%$ & 21 & $2.3 \%$ & 11 & $6.3 \%$ \\
\hline Larger truck & 98 & $8.2 \%$ & 4 & $3.1 \%$ & 33 & $3.7 \%$ & 61 & $35.1 \%$ \\
\hline
\end{tabular}

${ }^{*}$ Base category.

generalized ordered logit model to identify some factors associated with the injury severity of e-cyclists. The injury severity level is a discrete dependent variable, and a discrete choice model (e.g., logit or probit) is an appropriate method for modeling it [29-35].

When the severity category is $I(I \geq 3)$, the probability that a crash severity category $i$ occurs in an observed crash $n$ can be expressed as

$$
P_{n}(i)=P\left(U_{i n} \geq U_{j n}\right), \quad \forall j \neq i ; i, j \in I,
$$

where $P_{n}(i)$ is the probability that a crash severity category $i$ occurs in an observed crash $n, U_{i n}$ is a linear function that determines the severity of the crash $n$. As usual, $U_{\text {in }}$ can be linearly formed by

$$
U_{\text {in }}=\beta_{i} X_{n}+\varepsilon_{\text {in }},
$$

where $X_{n}$ is a vector of measurable characteristics (risk factors) that determine severity, $\beta_{i}$ is a vector of computable coefficients to be estimated, and $\varepsilon_{i n}$ is a disturbance term that takes into account the unobserved effects. One of the categories was normalized to zero in the discrete choice model since the measurable characteristics $\left(X_{n}\right)$ were fixed across severity categories. It means that at most $I-1$ categories can enter the model with $I$ levels of injury severity.

Assumed that the error term $\varepsilon_{i n}$ is generalized extreme value distributed independently, a multinomial logit (MNL) model can be derived to estimate the probability of a crash severity category with the expression

$$
P_{n}(i)=\frac{\exp \left(\beta_{i} X_{n}\right)}{\sum_{j} \exp \left(\beta_{j} X_{n}\right)}, \quad \forall j \neq i ; i, j \in I .
$$

In this case, the error terms $\left(\varepsilon_{i n}\right)$ are supposed to be independent in each of the severity categories. The regression coefficient $\left(\beta_{i}\right)$ in equation (3) can be estimated by the standard maximum likelihood estimation method. Before using the MNL model for analysis, it is necessary to set the reference category in advance. In addition to the IIA assumption, the MNL model also assumes that there is no difference between the sample individuals, that is, the effect of the variables in the model on the dependent variable is fixed.

However, studies have shown that the existence of heterogeneity may bias the estimation of model parameters and inference results [36,37]. Although the traditional fixed parameter model has been improved to characterize the heterogeneity, it should be accorded enough attention. Judging from the modeling methods cited by scholars, the RPL model is still the most widely used model in various fields. While investigating the factors that affect the severity of accidents, Kim et al. [38] developed a RPL model of driver injury severity in single-vehicle crashes, and then verified the individual heterogeneity based on age and gender. Zhou et al. [30] established a RPL model to estimate the likelihood of fatal and severe injuries on public buses in noncollision incidents. These relative studies [30-33, 37, 38] give support to the use of RPL models in injury severity research.

By allowing the parameters of all or part of the independent variables to vary across the observations, the RPL model can explain the unobserved heterogeneity in the data, and will not be limited by the "IIA" assumption. It is usually necessary to test the validity of the model in a regression analysis. The commonly used methods are the likelihood ratio test or chi-square test, which can be calculated as

$$
\chi^{2}=-2(L L(0)-L L(\beta)) \sim \chi^{2}(K)
$$

where $L L(0)$ is the initial value of the log-likelihood at zero, that is, the value of the log-likelihood when no independent variable is included in the model and only a constant term is included; $L L(\beta)$ is the convergence value of the log-likelihood function, that is, the value of the log-likelihood function when all significant independent variables and constant terms are included in the model; $K$ is the number of parameters in the model, $\chi^{2}(K)$ is the Chi-square value (critical value) for a given significance level $\alpha$. If the $\chi^{2}$ is greater than $\chi^{2}(K)$, it indicates that the model with added parameters is better than the model with only constant terms, which means that the model is effective. The Akaike Information Criterion (AIC), Bayesian Information Criterion (BIC), and McFadden's Pseudo $R^{2}$ are usually used to evaluate the fitness of a theoretical model. The calculation method of each evaluation index can be defined as

$$
\begin{aligned}
\mathrm{AIC} & =-2 L L(\beta)+2 K, \\
\mathrm{BIC} & =-2 L L(\beta)+\ln (N) \times K, \\
\text { Pseudo } R^{2} & =1-\frac{L L(\beta)}{L L(0)},
\end{aligned}
$$

where $N$ is the number of accidents. The smaller the value of the AIC and BIC indicators, the higher the fitness of the model; the McFadden's Pseudo $R^{2}$ value ranges from 0 to 1 . 
The closer the value is to 1 , the higher the fitness of the model.

The regression parameters obtained by using the MNL model can only qualitatively but not quantitatively reflect the impact of the significant independent variables on the accident outcomes. It means that the model parameters cannot be directly interpreted. In order to interpret the impact of each individual independent variable on the injury severity, normal derivatives of the injury severity probabilities are calculated. When the observed factor $X_{n m}$ changed, the change extent of the probability that cyclist $n$ is suffering severity $i$ can be expressed as

$$
\frac{\partial P_{n}(i)}{\partial X_{n m}}=\frac{\partial S_{i n}}{\partial X_{n m}} P_{n}(i)\left(1-P_{n}(i)\right)
$$

If the explanatory variable is an indicator variable, the pseudo-elasticity indicates that going from 0 to 1 may be more proper. The direct pseudo-elasticities can be computed to assess the effect of individual parameter estimates on cyclist injury severity probabilities as

$$
E_{X_{n m}}^{P_{n}(i)}=\left[\exp \left(\beta_{i m}\right) \frac{\sum_{I} \exp \left(A S C_{i}+\beta_{i} X_{n}\right)}{\sum_{I} \exp \left(\Delta\left(A S C_{i}+\beta_{i} X_{n}\right)\right)}-1\right] \times 100
$$

where $E_{X_{n m}}^{P_{n}(i)}$ is the direct pseudo-elasticity of the $m$-th variable from the vector $X_{n}, I$ is the number of possible severity categories, $A S C_{i}+\beta_{i} X_{n}$ is the value of the function determining the severity category when $X_{n m}$ equals zero, and $\Delta\left(A S C_{i}+\beta_{i} X_{n}\right)$ is the value after $X_{n m}$ has been changed from zero to one.

Some papers have explored the effects of sample size on the statistical fitness of random parameter models. According to studies by Ye and Lord [39, 40], when the sample size increases to the stable point, the estimated values become very close to the "true" values. Although the sample size of 1201 does not satisfy the requirement of minimum sample size for the RPL model, it is very close to the stable point. Also, the sample size is less than 5000 in many researches that have utilized the RPL models $[29,37,39,41-45]$. Considering the difficulty in collecting data about e-bike collisions, it was decided that a sample size of 1201 is suitable to build the RPL model for this study. The random parameter models still can provide a reasonable level of accuracy by using less detailed data [46].

\section{Results}

When the variables are correlated significantly, they should not be input into the model at the same time. The Variance Inflation Factor (VIF) between each pair of variables was calculated. The VIF value of all the selected variables in the models fell below 2, revealing no potential multicollinearity problem. The explanatory variables were selected by a stepwise procedure. During the modeling process, variables were retained in the specification if they had $t$-statistics corresponding to the $90 \%$ confidence level or higher on a two-tailed $t$-test.
Since PDO was the basic alternative category, only two severity categories (fatal and injury) entered the model. It should be noted that these results can only reveal the probability of injury severity resulted from crashes recorded in the videos. It did not reveal the probability of a crash occurrence or the crash frequency. The tests for model estimation of the discrete choice models were performed based on the commercial statistical software NLOGIT5.0. A simulation-based maximum likelihood method was utilized to estimate the parameters. The normal, lognormal, triangular, and uniform distributions that have been suggested in the literature for the random parameter models were considered. However, only the normal distribution was found to be statistically significant and provided the best statistical fit. This finding was consistent with previous studies where random parameters were considered [47]. Hence, the normal distribution was used in the RPL model. In addition, 500 Halton draws were utilized in the parameter estimation procedure since the efficiency of Halton draws was primarily significant compared to random draws. The random parameters were retained if their standard deviations had $t$-statistics corresponding to the $90 \%$ confidence level or higher. A forward stepwise procedure regression was applied for calibrating the traditional MNL model and the RPL model. Both of them have identified 20 significant factors at the $90 \%$ confidence level. Model comparison indicated that the RPL model is statistically superior to the MNL model. The parameter estimation results excluding the fixed-effects modeling results are shown in Table 2, including the loglikelihood, AIC and BIC.

The direct pseudo-elasticity effects for the RPL model are shown in Table 3. The two types of explanatory variables in the results are variables with standard fixed parameters and variables that produce statistically significant random parameters. An estimated parameter is verified to be random across cyclists when the standard deviation of the parameter density is statistically significant. The number of variables in the two types varies between different injury severity levels, which means that the proposed RPL model is appropriate for capturing the unobserved heterogeneities across individual cyclists.

The preliminary analyzing of RPL model results indicated that various important variables were significantly associated with the injury severity of cyclists, including collision avoidance and violation behaviors of drivers and cyclists, vehicle type, crash location, day of time, weather conditions, visibility restrictions. A positive coefficient value for an explanatory variable means it is positively associated with the injury severity level and increases the propensity of injury severity level with an increase in its magnitude. Our findings revealed no noticeable influence of some variables on the injury severity of cyclists, such as road type, crash type, and "Whether the cyclist has a lookout motion?." The five statistically significant standard deviations (random parameters) for the variables are shown in Table 2, which indicates that the probability of cyclists' suffering from fatal outcomes varied significantly across crashes. Both parameters as well as the 
TABLE 2: Coefficients (and standard errors) of the RPL model for cyclist injury severity outcomes.

\begin{tabular}{|c|c|c|c|}
\hline \multirow{2}{*}{ Characteristic } & \multirow{2}{*}{ Variable } & \multicolumn{2}{|c|}{ RPL } \\
\hline & & Injury & Fatal \\
\hline \multirow{4}{*}{ Collision avoidance behaviors of drivers } & Decelerating & $-0.494(0.247)^{* *}$ & $-6.351(1.688)^{* * *}$ \\
\hline & Std. dev. decelerating & - & $4.851(1.227)^{* * *}$ \\
\hline & Decelerating and lane change & - & $-3.787(1.560)^{* *}$ \\
\hline & Std. dev. decelerating and lane change & - & $7.252(2.149)^{* * *}$ \\
\hline \multirow{6}{*}{ Violation behaviors of drivers } & Speeding & - & $3.792(1.264)^{* * *}$ \\
\hline & Std. dev. speeding & - & $5.177(2.009)^{* * *}$ \\
\hline & Illegal lane change & $1.239(0.443)^{* * *}$ & - \\
\hline & Red-light violation & - & $5.341(2.266)^{* *}$ \\
\hline & Driving in the opposite direction & $1.409(0.746)^{*}$ & $5.987(1.667)^{* * *}$ \\
\hline & Illegal U-turn & $1.083(0.382)^{* * *}$ & - \\
\hline \multirow{4}{*}{ Collision avoidance behaviors of cyclists } & Accelerating & $-2.206(0.679)^{* * *}$ & - \\
\hline & Decelerating & $-1.168(0.257)^{* * *}$ & $-3.042(0.976)^{* * *}$ \\
\hline & Decelerating and lane change & $-0.703(0.250)^{* * *}$ & $-4.202(1.542)^{* * *}$ \\
\hline & Std. dev. decelerating and lane change & - & $4.087(1.732)^{* *}$ \\
\hline \multirow{3}{*}{ Violation behaviors of cyclists } & Speeding & - & $2.523(1.377)^{*}$ \\
\hline & Std. dev. speeding & - & $3.128(1.531)^{* *}$ \\
\hline & Red-light violation & $1.450(0.372)^{* * *}$ & $2.432(0.944)^{* *}$ \\
\hline \multirow{3}{*}{ Vehicle type } & Larger bus & - & $5.576(1.767)^{* * *}$ \\
\hline & Small truck & - & $5.615(1.635)^{* * *}$ \\
\hline & Larger truck & - & $11.160(2.533)^{* * *}$ \\
\hline Crash location & Curved & - & $-3.436(1.747)^{* *}$ \\
\hline Day of time & Nighttime & $1.392(0.369)^{* * *}$ & $3.590(0.953)^{* * *}$ \\
\hline Weather conditions & Cloudy & $1.296(0.467)^{* * *}$ & - \\
\hline \multirow{2}{*}{ Visibility restrictions } & Fixed obstacle & $1.664(0.392)^{* * *}$ & $1.889(1.019)^{*}$ \\
\hline & Moving vehicle & $0.541(0.244)^{* *}$ & - \\
\hline \multirow{6}{*}{ Constant } & $\mathrm{a} 1 / \mathrm{a} 2$ & $1.933(2.580)^{* * *}$ & \multirow{6}{*}{$-0.309(0.482)^{* *}$} \\
\hline & Log-likelihood & -648.025 & \\
\hline & Freedom & 34 & \\
\hline & AIC & 1364.0 & \\
\hline & $\mathrm{BIC}$ & 1537.1 & \\
\hline & McFadden's pseudo $R^{2}$ & 0.509 & \\
\hline
\end{tabular}

Note: Symbols ${ }^{*},{ }^{* *}$, and ${ }^{* * *}$ indicate significance at $10 \%, 5 \%$, and $1 \%$ level, respectively. Std. dev. $=$ standard deviation.

corresponding standard deviations listed in Table 2 were significant.

\section{Discussion}

6.1. Effects of Human Behaviors. With regard to the effect of collision avoidance behaviors on the injury severity outcomes, the parameters of driver decelerating, driver decelerating and lane change, and cyclist decelerating and lane change are all found to be random and statistically significant. These variables will reduce the likelihood of fatal crashes. The parameters of driver decelerating (specific to fatal) are random and have a mean of -6.351 and standard deviation of 4.851 . With these values, the normal distribution curve indicates that when the driver takes decelerating behaviors to avoid collision, most (90.48\%) of the cyclists will get a lower probability of sustaining a fatal outcome than without avoidance behaviors. However, about $9.52 \%$ of the cyclists will get a higher probability of sustaining a fatal injury. This can be attributed to the errors of few cyclists in stress response behaviors since those individuals may have a different experience in dealing with emergencies. That is, the result of the model is reasonable. The direct pseudo-elasticity effect of driver decelerating indicates that the probability of a cyclist sustaining a fatal outcome is about 51.33 percentage points lower than that of drivers without collision avoidance behaviors. Driver decelerating is also found to be statistically significant to reduce the likelihood of a cyclist sustaining an injury outcome by $2.86 \%$, but the effects defined for injury outcomes do not vary across crashes (the parameters are fixed). Similar results are also found for the cyclist accelerating, decelerating, decelerating and lane change that are defined for the injury level. The parameters are negative, which indicates that the likelihood of injury outcomes decreased by $0.24 \%, 1.90 \%$, and $2.11 \%$, respectively. These findings are generally consistent with expectations and previous studies [9].

In the following part, results of the random parameters are reported without the mean and standard deviation. Also, statements regarding the "rest of the crashes" are omitted since they can be deduced from the reported findings. The next significant random parameter for the normal condition model is "decelerating and lane change." The fatal outcome result indicates that, when the drivers take decelerating and 
TABLE 3: Elasticity analysis of the RPL model for cyclist injury severity outcomes.

\begin{tabular}{|c|c|c|c|c|}
\hline Characteristic & Variable & $\mathrm{PDO}$ & Injury & Fatal \\
\hline \multirow{2}{*}{ Collision avoidance behaviors of drivers } & Decelerating & 0.3012 & -0.0286 & -0.5133 \\
\hline & Decelerating and lane change & 0.2731 & 0.2712 & -0.2545 \\
\hline \multirow{5}{*}{ Violation behaviors of drivers } & Speeding & -0.0751 & -0.0863 & 0.9003 \\
\hline & Illegal lane change & -0.0061 & 0.0074 & -0.0007 \\
\hline & Red-light violation & -0.0176 & -0.0813 & 0.0808 \\
\hline & Driving in the opposite direction & -0.0831 & 0.0617 & 0.2588 \\
\hline & Illegal U-turn & -0.0084 & 0.0102 & -0.0942 \\
\hline \multirow{3}{*}{ Collision avoidance behaviors of cyclists } & Accelerating & 0.0252 & -0.0024 & 0.0220 \\
\hline & Decelerating & 0.1999 & -0.0190 & -0.4932 \\
\hline & Decelerating and lane change & 0.2004 & -0.0211 & -0.5156 \\
\hline \multirow{2}{*}{ Violation behaviors of cyclists } & Speeding & -0.0377 & -0.2451 & 0.3276 \\
\hline & Red-light violation & -0.0263 & 0.2427 & 0.4048 \\
\hline \multirow{3}{*}{ Vehicle type } & Larger bus & -0.0175 & -0.0523 & 0.0562 \\
\hline & Small truck & -0.0172 & -0.1331 & 0.1335 \\
\hline & Larger truck & -0.0006 & -0.7512 & 0.7880 \\
\hline Crash location & Curved & 0.0125 & 0.1711 & -0.1758 \\
\hline Day of time & Nighttime & -0.2233 & 0.0212 & 0.5458 \\
\hline Weather conditions & Cloudy & -0.0891 & 0.1092 & -0.0998 \\
\hline \multirow{2}{*}{ Visibility restrictions } & Fixed obstacle & -0.2506 & 0.0238 & 0.2695 \\
\hline & Moving vehicle & -0.1189 & 0.1133 & -0.1038 \\
\hline
\end{tabular}

lane change behaviors to avoid collision, about $69.92 \%$ of the cyclists will have a lower probability of sustaining a fatal injury than drivers without avoidance behaviors, with the direct pseudo-elasticity value of -0.2545 . When the cyclists take decelerating and lane change behaviors to avoid collision, about $84.81 \%$ of the cyclists will have a lower probability of sustaining a fatal outcome than cyclists without avoidance behaviors, with the direct pseudo-elasticity value of -0.0053 . The parameter of cyclist decelerating behaviors is -3.042 defined for fatal level, and the pseudoelasticity in Table 3 indicated that it reduces the likelihood of sustaining a fatal outcome by $49.32 \%$.

The estimated parameters of speeding behaviors by drivers and cyclists vary across crashes (the parameters are random). When the drivers have violation behaviors such as speeding, about $76.81 \%$ of the cyclists will get a higher probability of sustaining a fatal outcome than drivers without violation behaviors, and the direct pseudo-elasticity value across observations is 0.9003 . When the cyclists have violation behaviors such as speeding, about $79.00 \%$ of the cyclists will have a higher probability of sustaining a fatal outcome than cyclists without violation behaviors, with a pseudo-elasticity value of 0.3276 . This finding reflects the effects of speeding violation behaviors on aggravating the injury severity. Similar results were got by studies based on samples of e-bike riders in China [3, 8-10]. However, when it comes to the severity of riders, researchers have not reached a unanimous opinion. Severe injuries among e-bike riders were associated with speeding. What is more, researchers found that e-bike riders turning left and crossing the road in signalized intersections and curved roads had increased injury severity [8]. A three-year report of traffic crash data from China showed that $90 \%$ of fatal crashes were related to unsafe riding behaviors [48]. This result is intuitive in line with the laws of physics because the maximum speed of the electric bicycle itself is limited to a lower level, and the aggravation degree of accident injury severity caused by cyclists' speeding behaviors is less than that of drivers. The parameters of all violation behaviors by drivers and cyclists are positive, including illegal lane change, red-light violation, driving in the opposite direction, and illegal U-turn. These indicate that they have increased the injury severity to a certain extent. This result is in alignment with previous research [10, 49, 50]. Riding in motor vehicle lanes is a common reason for e-bike-related crashes [13]. In view of the difficulty in obtaining exact information on driver's precrash behaviors, these factors are not included in other studies about the levels of accident injury severity.

In the developing countries, the traffic management departments have not focused enough attention to electric bicycles, and law enforcement for electric bicycles is rare. This background brings about unsafe conditions to road occupants in the major cities. According to the results of elasticity effects, the violation behaviors of cyclists are key factors that increase the probability of fatal outcomes. The most effective measurement of improving the safety conditions related to electric bicycles is to reduce the occurrence of speeding behaviors. Violations (risky behaviors) will increase the likelihood of sustaining more serious outcomes. It is consistent with previous findings $[10,13-15]$. The authorities need to strengthen the enforcement of laws and then force people to abide by traffic rules. Zero tolerance for traffic violations related to electric vehicles (e.g., speeding and red-light violation) needs to be supplemented with cyclist education on road traffic crashes. Education by completing training courses for cyclists needs to be promoted to prevent them from being injured in accidents.

It is worth noting that this high-frequency illegal behavior will not only hurt electric bicycle riders but also pose a 
threat to the safety of life and property of other road users [51]. With technology development, China has installed stable and reliable traffic surveillance cameras to monitor traffic violations. Even if the behavior of running a red light does not always cause a crash, riders will be fined for the negative impact of disturbing traffic flow. Although riders are generally aware of safe driving behaviors such as obeying traffic rules, they still violate traffic rules and show unsafe behaviors [52]. Therefore, in addition to enhancing safety awareness, more campaigns should also be conducted to inform cyclists that cameras have been installed to monitor their compliance with the law.

Therefore, carrying out educational activities to increase traffic safety awareness and introduce traffic laws may be an effective way to reduce crashes. It is supplemented by increasing the driver's awareness of cyclists and educating them about sharing road infrastructure with electric bicycles. For instance, law enforcement agencies and local agencies can distribute some handouts to traffic occupants. Advertising can convey simple and effective information, and media events can help publicize law enforcement. More general public education through both traditional and social media about improved hazard avoidance at lower speeds and the dangers associated with swerving can also be incorporated into road safety information programs.

6.2. Effects of Vehicle Characteristics. The specific types of vehicles (large trucks, passenger cars, etc.) may be more dangerous for cyclists. Relative to the passenger car, the parameter for fatal level of larger bus, smaller truck, and larger truck are 5.576, 5.615, and 11.160, respectively. It shows that these three types of vehicles will significantly increase the probability of fatal outcomes by $5.62 \%, 13.35 \%$, and $78.80 \%$, respectively. Study [53] found that for crashes colliding with the truck, the likelihood of riders suffering from severe injury increases by $9.5 \%$ on average. The reason may be related to the characteristics of large vehicles such as heavy weight, and also related to the obvious visual blind spots. Under the same conditions, the collision safety performance of large vehicles is better, but this is at the expense of other vehicles' safety. Therefore, when the electric bicycle collides with a large vehicle, the cyclist is more likely to suffer serious injuries. Some other previous studies [7, 33] also concluded similar findings. In some areas, in order to reduce the damage of larger trucks to cyclists and give the driver and cyclist enough time to observe, it is stipulated that when driving on urban roads, the driver need to stop for a while before turning right. The cyclist also needs to understand the basic knowledge of possible visual blind spots in large vehicles.

In a study on electric bicycle accidents [4], the crash type was found to be one of the most important predictors of the severity of bicycle crashes. In angle and side crashes, the likelihood of sustaining fatalities were lower for cyclists than injury category. Some studies on the severity of vehicle accidents have focused on the specific crash type (such as head-on, rear-end, single-vehicle, etc.), and found that the crash type was an important factor affecting the accident outcome. However, based on the data used in this paper, sufficient evidence cannot be obtained to show that the crash type of vehicle-electric bicycle crash has a significant impact on the injury level of cyclists.

6.3. Effects of Roadway Attributes. The crash location was found to be the key factor of injury severity outcomes for truck crashes. Similarly, it may help to indicate the cyclist injury severity. Table 2 also shows that crashes occurring on the curved segments tend to be less severe (as indicated by elasticity values) with $17.58 \%$ lower probability of fatal outcomes relative to straight segments. The possible reason for this finding is that the speed of vehicles may have decreased before entering the curve segments, thereby reducing the risk of injury caused by collision kinetic energy. In the straight section, vehicles often run at high speeds and drivers are not alert enough. There is no sufficient evidence to prove that road type is an important factor affecting injury severity. For the factor of road type, previous related studies did not get a consistent conclusion in whether it is significantly related with the dependent variable $[54,55]$.

6.4. Effects of Environmental Conditions. The day of time was labeled as nighttime and daytime. The significant coefficients of injury and fatal outcomes at nighttime are 1.392 and 3.590 , respectively. Indicating that compared with daytime, the probabilities of being injured and fatal outcomes are found to be $2.12 \%$ and $54.58 \%$ higher for cyclists who are involved in crashes that occurred at nighttime. This may be attributed to the dim light at nighttime, which can potentially affect the perception-reaction time of the driver and cyclist. Most crashes that happened at nighttime were found to be without the presence of street lightings [48]. Helmet use was not optimal in the daytime. Also, drivers may drive faster in nighttime [19]. These may well explain some conclusions of this study.

Compared with sunny conditions, the probability of injury outcomes is found to be $10.92 \%$ higher for cyclists who are involved in crashes that occurred in cloudy conditions. In the studies about injury severity of truck-involved, single-vehicle, or rear-end crashes $[28,47,49]$, the weather conditions were also identified as a valuable factor to predict the injury severity level.

The vision of drivers and cyclists are often occluded by some obstacles. The results show that when the visibility is restricted by a fixed obstacle, the probability of injury and fatal results are significantly increased. While the visibility is restricted by a running vehicle, it will significantly increase the probability of injury outcomes. Since the information about whether the restrictions exist or not is very difficult to obtain, it is rarely considered in other studies about crash injury severity. Similarly, the factor of "electric bicycle visibility time" can only be determined by the circumstances prior to the accident. Fortunately, we take these factors into account through the data obtained from the video. 


\section{Conclusions}

This study takes vehicle-electric bicycle collisions as a sample to analyze the main factors related to cyclist injury severity. Our investigation aims to propose some countermeasures to alleviate traffic accidents injury damage in the city. The results show that the significant factors are precrash behaviors, vehicle type, crash location, and environmental conditions. While some variables conform to previous studies, other variables offer new insights into the cyclist injury severity. Overall, the fitness of the RPL model appears to be reasonable, but the confidence intervals around most covariates were large, suggesting imprecision in the parameters that we recorded and measured. This implies that the response of drivers was variable, relative to the conditions represented in the video. That imprecision is likely magnified by the relatively small sample size that we assessed. As the number of videos increases, our model will further improve its ability to discover other important factors related to severity.

Since it is unrealistic to prohibit the use of electric bicycles in China, laws and rules need to be strengthened so that effective regulations can be formulated. This study contributed to the field of research by developing a RPL model to examine the impacts of various factors on injury severity across fatal, injury, and PDO crashes involving e-cyclists in China, especially concluded factors about precrash behaviors of drivers and cyclists. The unique information about visibility restrictions and "electric bicycle visibility time" were also extracted from the video. Based on these findings, our countermeasures include zero tolerance for e-cyclists-related traffic violations and raising awareness about e-cyclist safety. It is also necessary to increase investment in road conditions, but education on road safety culture is the key. As a result, we believe that changing the cycling culture of e-cyclists requires tremendous effort-the education and law enforcement are key elements.

Finally, some limitations need to be addressed. Firstly, this study encountered several data limitations and differences. Regarding the study of traffic accident data, the previous researches were mainly focused on the severity and frequency of accident injuries. The data in this article come from the video, and only divide the injury severity into three categories, so it cannot cover a wider range of crash severity. The aspect that affects the frequency of accidents is not considered, and the study of the frequency of accidents can provide a theoretical basis for identifying the accident-prone sections and optimizing road design. Therefore, in the future research, we should strengthen the analysis of the factors that affect the frequency of accidents while conducting the research on the severity of accidents, and conduct a comprehensive research on traffic accident data.

Secondly, our data suffer from underreporting of PDO crashes, which may affect the accuracy. We only observed a specific cross-sectional data with small sample size, that is to say, we analyzed the specific conditions under which the collision occurred. This is not enough to represent the national situation. Future work needs to be undertaken with a wider use of databases, including crashes after 2019 and cases in other parts of China.

In addition, the data from videos are used for analysis, and the influence of individual demographic characteristics on the results is not considered, even though most of the results of this study are consistent with the previous studies on the analysis of electric-bicycle-related accidents. Moreover, traffic accidents are caused by multiple complex factors, including driver characteristics, vehicle conditions, road conditions, and traffic and environmental conditions. However, due to the lack of data content, some other variables that may affect the cyclist injury degree were not included in the model, such as the speed of the vehicle at the time of the accident, the initiation of the airbag, whether the driver wears a seat belt, whether to use a mobile phone, and whether to take avoidance measures. In future research, the natural driving data may be used to comprehensively explore the influence mechanism of risk factors on accident severity.

We did not use more complex models (e.g., multivariate counting models) to analyze the frequency of crashes under various severity levels. Although the RPL model partially solved the unobserved heterogeneity problem in the data, the traffic accident data itself may have problems such as spatial heterogeneity. Methods such as machine learning and artificial neural networks can be used to optimize the existing statistical models, so as to provide new perspectives and directions for traffic safety research. Nevertheless, this research still provides a foundation for the transportation management of developing countries to develop effective countermeasures against accidents involving electric bicycles.

\section{Data Availability}

The data used to support the findings of this study are available from the corresponding author upon request.

\section{Conflicts of Interest}

The authors declare that they have no conflicts of interest.

\section{Acknowledgments}

This research was jointly supported by the National Natural Science Foundation of China (51178054 and 51978069).

\section{References}

[1] S. Will and E. A. Schmidt, "Powered two wheelers' workload assessment with various methods using a motorcycle simulator," IET Intelligent Transport Systems, vol. 9, no. 7, pp. 702-709, 2015.

[2] Z. Feng, R. P. Raghuwanshi, Z. Xu, D. Huang, C. Zhang, and T. Jin, "Electric-bicycle-related injury: a rising traffic injury burden in China," Injury Prevention, vol. 16, no. 6, pp. 417-419, 2010.

[3] Q. Yuan, H. Yang, J. Huang, S. Kou, Y. Li, and A. Theofilatos, "What factors impact injury severity of vehicle to electric bike crashes in China?" Advances in Mechanical Engineering, vol. 9, no. 8, pp. 1-10, 2017. 
[4] P. Hertach, A. Uhr, S. Niemann, and M. Cavegn, "Characteristics of single-vehicle crashes with e-bikes in Switzerland," Accident Analysis \& Prevention, vol. 117, pp. 232-238, 2018.

[5] C. Cha Sow King, M. Liu, S. Patel, T. Goo, W. Lim, and H. Toh, "Injury patterns associated with personal mobility devices and electric bicycles: an analysis from an acute general hospital in Singapore," Singapore Medical Journal, vol. 61, no. 2, pp. 96-101, 2020.

[6] W. Du, J. Yang, B. Powis et al., "Epidemiological profile of hospitalised injuries among electric bicycle riders admitted to a rural hospital in Suzhou: a cross-sectional study," Injury Prevention, vol. 20, no. 2, pp. 128-133, 2014.

[7] F. Hu, D. Lv, J. Zhu, and J. Fang, "Related risk factors for injury severity of e-bike and bicycle crashes in Hefei," Traffic Injury Prevention, vol. 15, no. 3, pp. 319-323, 2014.

[8] T. Wang, J. Chen, C. Wang, and X. Ye, "Understand e-bicyclist safety in China: crash severity modeling using a generalized ordered logit model," Advances in Mechanical Engineering, vol. 10, no. 6, pp. 1-11, 2018.

[9] L. Hu, X. Hu, J. Wang, A. Kuang, W. Hao, and M. Lin, "Casualty risk of e-bike rider struck by passenger vehicle using China in-depth accident data," Traffic Injury Prevention, vol. 21, no. 4, pp. 283-287, 2020.

[10] Y. Qian, Q. Sun, G. Fei et al., "Riding behavior and electric bike traffic crashes: a Chinese case-control study," Traffic Injury Prevention, vol. 21, no. 1, pp. 24-28, 2020.

[11] S. Edge, J. Dean, M. Cuomo, and S. Keshav, "Exploring e-bikes as a mode of sustainable transport: a temporal qualitative study of the perspectives of a sample of novice riders in a Canadian city," The Canadian Geographer/Le Géographe Canadien, vol. 62, no. 3, pp. 384-397, 2018.

[12] A. Fyhri, O. Johansson, and T. Bjørnskau, "Gender differences in accident risk with e-bikes-survey data from Norway," Accident; Analysis and Prevention, vol. 132, Article ID 105248, 2019.

[13] C. Ma, D. Yang, J. Zhou, Z. Feng, and Q. Yuan, "Risk riding behaviors of urban e-bikes: a literature review," International Journal of Environmental Research and Public Health, vol. 16, no. 13, 2019.

[14] X. Zhang, Y. Yang, J. Yang et al., "Road traffic injuries among riders of electric bike/electric moped in southern China," Traffic Injury Prevention, vol. 19, no. 4, pp. 417-422, 2018.

[15] Y. Guo, J. Zhou, Y. Wu, and J. Chen, "Evaluation of factors affecting e-bike involved crash and e-bike license plate use in China using a bivariate probit model," Journal of Advanced Transportation, vol. 2017, Article ID 2142659, 12 pages, 2017.

[16] S. A. Zhou, A. F. W. Ho, M. E. H. Ong et al., "Electric bicyclerelated injuries presenting to a provincial hospital in China: a retrospective study," Medicine, vol. 96, no. 26, Article ID e7395, 2017.

[17] C. Wang, C. Xu, J. Xia, and Z. Qian, "The effects of safety knowledge and psychological factors on self-reported risky driving behaviors including group violations for e-bike riders in China," Transportation Research Part F: Traffic Psychology and Behaviour, vol. 56, pp. 344-353, 2018.

[18] T. Weber, G. Scaramuzza, and K.-U. Schmitt, "Evaluation of e-bike accidents in Switzerland," Accident Analysis \& Prevention, vol. 73, pp. 47-52, 2014.

[19] T. Champahom, S. Jomnonkwao, D. Watthanaklang, A. Karoonsoontawong, V. Chatpattananan, and V. Ratanavaraha, "Applying hierarchical logistic models to compare urban and rural roadway modeling of severity of rear-end vehicular crashes," Accident Analysis \& Prevention, vol. 141, Article ID 105537, 2020.
[20] G. Batouli, M. Guo, B. Janson, and W. Marshall, "Analysis of pedestrian-vehicle crash injury severity factors in Colorado 2006-2016," Accident Analysis \& Prevention, vol. 148, Article ID 105782, 2020.

[21] H. Naci, D. Chisholm, and T. D. Baker, "Distribution of road traffic deaths by road user group: a global comparison," Injury Prevention, vol. 15, no. 1, pp. 55-59, 2009.

[22] Y. Guo, A. Osama, and T. Sayed, "A cross-comparison of different techniques for modeling macro-level cyclist crashes," Accident Analysis \& Prevention, vol. 113, pp. 38-46, 2018.

[23] D. Ito, K. Hayakawa, Y. Kondo et al., "Difference between carto-cyclist crash and near crash in a perpendicular crash configuration based on driving recorder analysis," Accident Analysis \& Prevention, vol. 117, pp. 1-9, 2018.

[24] R. V. Rea, C. J. Johnson, D. A. Aitken, K. N. Child, and G. Hesse, "dash cam videos on youtube offer insights into factors related to moose-vehicle collisions," Accident Analysis \& Prevention, vol. 118, pp. 207-213, 2018.

[25] Y. Guo, T. Sayed, and M. H. Zaki, "Evaluating the safety impacts of powered two wheelers on a shared roadway in China using automated video analysis," Journal of Transportation Safety \& Security, vol. 11, no. 4, pp. 414-429, 2018.

[26] K. Min and A. Ando, "Analysis on characteristics of dangerous driving events via recorded data of drive-recorder," Transportation Research Procedia, vol. 48, pp. 1342-1363, 2020.

[27] V. Ratanavaraha and S. Suangka, "Impacts of accident severity factors and loss values of crashes on expressways in Thailand," IATSS Research, vol. 37, no. 2, pp. 130-136, 2014.

[28] A. K. Çelik and E. Oktay, "A multinomial logit analysis of risk factors influencing road traffic injury severities in the erzurum and kars provinces of Turkey," Accident Analysis \& Prevention, vol. 72, pp. 66-77, 2014.

[29] J. Wang, H. Huang, P. Xu, S. Xie, and S. C. Wong, "Random parameter probit models to analyze pedestrian red-light violations and injury severity in pedestrian-motor vehicle crashes at signalized crossings," Journal of Transportation Safety \& Security, vol. 12, no. 6, pp. 818-837, 2019.

[30] H. Zhou, C. Yuan, N. Dong, S. C. Wong, and P. Xu, "Severity of passenger injuries on public buses: a comparative analysis of collision injuries and non-collision injuries," Journal of Safety Research, vol. 74, pp. 55-69, 2020.

[31] M. Waseem, A. Ahmed, and T. U. Saeed, "Factors affecting motorcyclists' injury severities: an empirical assessment using random parameters logit model with heterogeneity in means and variances," Accident Analysis \& Prevention, vol. 123, pp. 12-19, 2019.

[32] Z. Li, Q. Wu, Y. Ci, C. Chen, X. Chen, and G. Zhang, "Using latent class analysis and mixed logit model to explore risk factors on driver injury severity in single-vehicle crashes," Accident Analysis \& Prevention, vol. 129, pp. 230-240, 2019.

[33] C. Fangrong, X. Pengpeng, Z. Hanchu, H. S. C. Alan, and H. Huang, "Investigating injury severities of motorcycle riders: a two-step method integrating latent class cluster analysis and random parameters logit model," Accident Analysis \& Prevention, vol. 131, pp. 316-326, 2019.

[34] M. Fanyu, X. Pengpeng, S. Cancan, G. Kun, Z. Zichu, and L. Yang, "Influential factors associated with consecutive crash severity: a two-level logistic modeling approach," International Journal of Environmental Research and Public Health, vol. 17, no. 15, 2020.

[35] F. Chen, M. Song, and X. Ma, "Investigation on the injury severity of drivers in rear-end collisions between cars using a random parameters bivariate ordered probit model," 
International Journal of Environmental Research and Public Health, vol. 16, no. 14, 2019.

[36] F. L. Mannering, V. Shankar, and C. R. Bhat, "Unobserved heterogeneity and the statistical analysis of highway accident data," Analytic Methods in Accident Research, vol. 11, pp. 1-16, 2016.

[37] Y. Guo, Z. Li, Y. Wu, and C. Xu, "Exploring unobserved heterogeneity in bicyclists' red-light running behaviors at different crossing facilities," Accident Analysis \& Prevention, vol. 115, pp. 118-127, 2018.

[38] J.-K. Kim, G. F. Ulfarsson, S. Kim, and V. N. Shankar, "Driverinjury severity in single-vehicle crashes in California: a mixed logit analysis of heterogeneity due to age and gender," $A c$ cident Analysis \& Prevention, vol. 50, pp. 1073-1081, 2013.

[39] F. Ye and D. Lord, "Comparing three commonly used crash severity models on sample size requirements: multinomial logit, ordered probit and mixed logit models," Analytic Methods in Accident Research, vol. 1, pp. 72-85, 2014.

[40] F. Ye and D. Lord, "Investigation of effects of underreporting crash data on three commonly used traffic crash severity models," Transportation Research Record: Journal of the Transportation Research Board, vol. 2241, no. 1, pp. 51-58, 2011.

[41] W. Wang, Z. Yuan, Y. Liu, X. Yang, and Y. Yang, "A random parameter logit model of immediate red-light running behavior of pedestrians and cyclists at major-major intersections," Journal of Advanced Transportation, vol. 2019, Article ID 2345903, 13 pages, 2019.

[42] W. Yin, P. Murray-Tuite, S. V. Ukkusuri, and H. Gladwin, "Modeling shadow evacuation for hurricanes with randomparameter logit model," Transportation Research Record: Journal of the Transportation Research Board, vol. 2599, no. 1, pp. 43-51, 2016.

[43] H. Wen and Z. Tang, "Comparative study on motorcycle crash injury severity estimation based on nested logit and random parameters logit models," Journal of South China University of Technology (Natural Science), vol. 46, no. 11, pp. 83-91, 2018.

[44] M. M. Hamed and B. M. Al-Eideh, "An exploratory analysis of traffic accidents and vehicle ownership decisions using a random parameters logit model with heterogeneity in means," Analytic Methods in Accident Research, vol. 25, pp. 100-116, 2020.

[45] N. A. Khan and M. A. Habib, "Understanding variations in activity-based vehicle allocation decisions: a latent segmentation-based random parameter logit modeling approach," Transportation Research Procedia, vol. 48, pp. 1505-1525, 2020.

[46] P. C. Anastasopoulos and F. L. Mannering, "An empirical assessment of fixed and random parameter logit models using crash- and non-crash-specific injury data," Accident Analysis \& Prevention, vol. 43, no. 3, pp. 1140-1147, 2011.

[47] A. Behnood and F. Mannering, "The effect of passengers on driver-injury severities in single-vehicle crashes: a random parameters heterogeneity-in-means approach," Analytic Methods in Accident Research, vol. 14, pp. 41-53, 2017.

[48] L. Lu, C. Wang, and T. Wang, "Improving e-bike safety on urban highways in China," Discrete Dynamics in Nature and Society, vol. 2015, Article ID 415237, 8 pages, 2015.

[49] C. Ma, W. Hao, W. Xiang, and W. Yan, "The impact of aggressive driving behavior on driver-injury severity at highway-rail grade crossings accidents," Journal of Advanced Transportation, vol. 2018, Article ID 9841498, 10 pages, 2018.

[50] N. Casado-Sanz, B. Guirao, and M. Attard, "Analysis of the risk factors affecting the severity of traffic accidents on
Spanish crosstown roads: the driver's perspective," Sustainability, vol. 12, no. 6, 2020.

[51] X. Gao, J. Zhao, and H. Gao, "Red-light running behavior of delivery-service e-cyclists based on survival analysis," Traffic Injury Prevention, vol. 21, no. 8, pp. 558-562, 2020.

[52] Z. Wang, R. L. Neitzel, X. Xue, W. Zheng, and G. Jiang, "Awareness, riding behaviors, and legislative attitudes toward electric bikes among two types of road users: an investigation in Tianjin, a municipality in China," Traffic Injury Prevention, vol. 20, no. 1, pp. 72-78, 2019.

[53] Z. Wang, S. Huang, J. Wang, D. Sulaj, W. Hao, and A. Kuang, "Risk factors affecting crash injury severity for different groups of e-bike riders: a classification tree-based logistic regression model," Journal of Safety Research, vol. 76, pp. 176-183, 2021.

[54] Y. Xing, Z. Sun, and D. Wang, "Investigating influence factors on injury severity of electric and non-electric bicycle crashes in Beijing," in Proceedings of the IEEE 5th International Conference on Intelligent Transportation Engineering (ICITE), pp. 606-610, Beijing, China, September 2020.

[55] W. Liyang, L. Ruimin, W. Changjun, and L. Zhiyong, "Driver injury severity analysis of crashes in a western China's rural mountainous county: taking crash compatibility difference into consideration," Journal of Traffic and Transportation Engineering, 2020, In press. 